\title{
Michèle FOGEL
}

\section{Les entrées royales françaises au prisme de l'échange}

Pour les chercheurs intéressés par ces manifestations complexes que sont les entrées royales françaises, l'année 2001 fut particulièrement faste : fruits des travaux du Groupe de recherche sur les entrées solennelles des villes françaises à la Renaissance (1484-1615) menés à l'Université Concordia par Marie-France Wagner et ses collègues, ont paru coup sur coup Le roi dans la ville. Anthologie des entrées royales dans les villes francaises de province (1615-1660), textes introduits et annotés par MarieFrance Wagner et Daniel Vaillancourt, chez H. Champion, Les arts $d u$ spectacle dans la ville (1404-1721), textes édités et présentés par MarieFrance Wagner et Claire Le Brun-Gouanvic, chez le même éditeur, et le numéro spécial $\left(\mathrm{n}^{\circ} 212\right)$ de la revue XVII siècle, sous le titre Les entrées royales.

Dans l'introduction de ce numéro, Marie-France Wagner et Daniel Vaillancourt affirmaient qu'au delà des études antérieures intéressées par la théâtralité et la magnificence, l'entrée était «reconfigurée pour ce qu'elle est : une pragmatique du pouvoir, un coup de force contre les autorités locales, un silence imposé à même les stratégies encomiastiques et la rhétorique jésuite » (p. 380-1). L'entrée paraissait ainsi s'être figée comme un moment de la relation forcément dissymétrique entre un roi conquérant et ses sujets.

La voici maintenant remise en mouvement, entraînée dans « Les jeux de l'échange ». L'échange, qu'on pourrait définir ici comme un rapport de sociabilité fondé sur la réciprocité des dons, devient le nouveau prisme au travers duquel sont considérées les rencontres non seulement entre rois et villes, mais aussi entre auteurs, acteurs et spectateurs d'un théâtre en formation, et encore, l'invention et la circulation de petites formes musicales ou littéraires offertes gracieusement à des particuliers ou au public. Point de vue fécond, on le voit. 
Plutôt que d'entreprendre le compte rendu des quinze articles que l'on trouve parfaitement décrits dans l'avant-propos (p. 9-12), je m'attacherai à examiner ceux qui traitent de l'entrée comme don et du don dans l'entrée et qui croisent le récent livre de Natalie Zemon Davis, L'esprit du don dans la France du XVT siècle.

L'ouvrage pionnier de Bernard Guenée et Françoise Lehoux, Les entrées royales françaises de 1328 à 1515, avait inscrit les entrées dans le cadre institutionnel de négociations inévitables, un «dialogue» disaient-ils, entre souverains et municipalités. Cette fois, il ne s'agit plus tant de dialogue que de biens matériels montrés, offerts, consommés. Jacky Provence dresse dans Les jeux de l'échange (p. 141-164) ce qu'il appelle joliment "la comptabilité de l'éphémère », celle des entrées à Troyes d'Henri IV en 1595 et de Louis XIII en 1629. Plus sèchement, plus précisément aussi que les livrets commémoratifs, les sources comptables permettent de suivre la mobilisation de la municipalité et des artisans dans la préparation de la réception : choix et mise en œuvre en un véritable chantier des matériaux de la décoration urbaine, 19,7\% des dépenses en 1595 et 26,7 en 1629; fabrication de vêtements de soie brodés des notables et du dais royal ainsi que des costumes des figurants des tableaux vivants, $24 \%$ en 1595 et 22,8 en 1629; description et valeur des présents au roi et à sa suite, respectivement 26,9 et 27,6\%. Ces proportions relativement inchangées d'une entrée à l'autre confirment le souci premier de la ville et de ses notables de se parer pour l'occasion, l'ensemble des parures étant lui-même considéré comme faisant partie du don. Parmi les présents proprement dits, on observe un glissement de la part des produits en nature, essentiellement le vin, vers celle des objets d'orfèvrerie précieux; la clé et le cœur d'or figurent toujours, mais ne sont pas particulièrement dispendieux. Les officiers royaux reçoivent aussi leur part, considérée comme la contrepartie du droit qu'ils ont sur les décorations, le dais et le cheval fourni au roi, souvenir de temps plus anciens et plus durs où il s'agissait de racheter leur droit de pillage.

Avec des revenus annuels inchangés, environ 12000 livres tournois, l'ensemble des frais engagés à Troyes s'élève en 1595 à 17078 livres et à 22886 en 1629. S'il est entendu que ce don doit immanquablement attirer le contre-don royal, celui-ci n'a rien de mécanique, ni 
d'équivalent, le plus souvent borné à la confirmation des droits particuliers de la ville, ses privilèges.

C'est bien ce que Bruno Paradis et Lise Roy constatent : "L'efficacité du don et les retombées politiques concrètes à court, moyen et long termes, sont très difficiles à cerner puisque jamais inscrites explicitement dans les archives» (p. 115). Avec les registres de délibérations et de comptabilité d'un corpus de villes étendu, ils présentent une lecture de l'entrée au XVI ${ }^{\mathrm{e}}$ siècle où se joignent l'intérêt pour ses aspects formels, compris comme « ritualisation dramatique du don » (p. 108), et une attention nouvelle à son contenu émotionnel, compris comme manifestation de cet amour politique étudié par Jean Nagle dans La civilisation du cour.

Du premier point, au delà de la déclinaison des étapes du rituel l'accueil de l'hôte devant les murs, la présentation et les harangues des corps de notables, la remise des clés, la progression dans la ville aux milieux des décorations triomphales, la messe suivie d'un Te Deum, le banquet -, je retiendrai la notion de «sacrifice contraint»: l'obligation pour la ville de dépenser apparemment sans compter, «libéralement», comme le roi et ses serviteurs sont réputés le faire, comme les nobles, et comme les villes rivales. En 1533, l'angoisse saisit les consuls de Romans en Dauphiné lorsqu'ils apprennent la venue de François $I^{\text {er }}$ et de sa famille alors que les villes voisines de Vienne et de Valence ont déjà établi leur dispositif. La même année, les consuls de Nîmes s'informent des villes du Languedoc et ne veulent rien livrer de leurs préparatifs. Commencent alors les manipulations financières, les emprunts inévitables et souvent forcés, voire l'établissement d'un nouveau prélèvement et, dans le cas de Nîmes, son extension à la région environnante.

Contrairement à l'exemple de Troyes, les comptes des villes prises à témoin pour le $\mathrm{XVI}^{\mathrm{e}}$ siècle montrent dans l'ensemble une plus grande part des présents au roi : sur onze cas, trois vont de 10,5 à $22 \%$, mais deux de 22 à 32, et six se situent entre 40 et $69 \%$ de l'ensemble des dépenses. C'est ce qui rend plus concret peut-être cette notion d'amour entre le roi et ses sujets présente dans les lettres royales qui 
annoncent sa venue, dans les harangues qu'il entend, dans les décorations qui l'accompagnent sur son parcours : au banquet, des jeunes filles somptueusement vêtues lui offrent des cœurs d'or ouvragés, et le lendemain, dans son logis, les notables à genoux lui présentent des objets précieux. Dans cette dernière phase, soustraite à la vue de la population, se font les demandes de nouveaux droits et, parfois, les agréments. Au delà, on trouve les dons en nature, nourritures et chevaux, offerts au roi, à sa famille et à sa suite.

Le don, dans sa ritualisation par l'entrée, se révèle ainsi comme signe d'appartenance au système monarchique et peut être réemployé pour accueillir les membres de la famille royale, les grands officiers et, bien entendu, les souverains étrangers de passage.

En suivant le jeune Louis XIV en Roussillon lorsqu'il va chercher son épouse à Saint-Jean-de-Luz en 1660, Fabrice Charton montre comment les consuls de Perpignan, la capitale de cette province de rattachement récent, proposent en vain d'organiser une entrée solennelle "conformément a celle de l'empereur Charles Quint» (p. 69). Le roi n'a nulle envie de se présenter comme le successeur légitime de l'empereur-roi de toutes les Espagnes. D'ailleurs, il pleut comme il peut pleuvoir en avril dans cette région: sans quitter son carrosse, il se fait conduire à l'église principale où il prie devant le Saint Sacrement, puis entend le Te Deum. Une conduite de conquérant qu'il confirme le lendemain en visitant la citadelle et les fortifications et en soupant le jour suivant chez le gouverneur du Roussillon qu'il a nommé. Dès lors, les notables n'ont de cesse de retrouver les voies de la dépense pour enclencher le processus du don et de sa contrepartie : un bal à l'hôtel de ville, la comédie dans la maison où loge la reinemère, du vin de muscat et des chandeliers, des citrons doux et des oranges. Les divertissements sont pauvres, les présents maigres : tout ce que le roi accorde aux notables, c'est de l'accompagner en cortège lorsqu'il va entendre la messe dans un couvent le deuxième jour puis à l'église principale le jour de son départ. Du moins a-t-il ainsi confirmé la place de chacun dans la ville. 
Les divertissements en viennent-ils à remplacer les cérémonies? Fabrice Charton semble le croire lorsqu'il suit la série des fêtes données en l'honneur des événements heureux de la vie du roi et de sa famille jusqu'au début du XVIII ${ }^{\mathrm{e}}$ siècle. C'est oublier que ces fêtes, qu'elles soient commandées par le roi ou par les autorités locales, s'inscrivent dans la trame désormais serrée des ordres royaux de célébration des Te Deum ${ }^{1}$.

Avec « Prestige et urbanité : le luxe dans la rue », Daniel Vaillancourt a voulu étendre les usages et significations de la dépense. Reprenant l'hypothèse de la ville laboratoire qui sous-tendait en partie les travaux parus en 2001, il fait de la ville parée pour l'entrée, en l'occurrence Rouen en 1550 et 1596, la scène nécessaire d'une prodigalité jusque-là aristocratique qu'à la suite d'Henri Baudrillart comme de Gilles Lipovetsky et Elyette Roux, il désigne sous le nom de luxe. Pour tous ceux qui entreprennent de déchiffrer les traces laissées par les morts, la question des intermédiaires se pose toujours : pour ma part, je préfère lire Nicolas Delamare, commissaire au Châtelet sous le règne de Louis XIV : «La magnificence diffère du luxe, en ce qu'elle ne s'écarte jamais de la droite raison, \& des règles de la bienséance : si les Princes \& les Grands font des dépenses splendides, paroissent avec pompe, cela est toujours proportionné à leur élévation et à leur revenu... donc c'est une vertu. [...] Le luxe au contraire n'a d'autres bornes que celle de l'ambition \& de la vanité..., c'est donc un vice. » (t. I, p. 413) Un vice parce que c'est une tentative individuelle de transgresser l'ordre social et ses codes de conduite.

Pour Daniel Vaillancourt, l'entrée accomplit la transgression collective d'un de ces codes exprimé dans la série des lois somptuaires édictées par la monarchie depuis la fin du $\mathrm{XV}^{\mathrm{e}}$ siècle. Contrairement à ce qu'il affirme, ces lois ont dès 1485 et à la demande des États Généraux euxmêmes un but premier de défense sociale et non économique : la dépense n'est superflue et excessive que lorsqu'elle prétend permettre

\footnotetext{
${ }^{1}$ La mise en place du système qui lie événement, lettre de cachet aux prélats et aux divers corps constitués ordonnant la célébration d'un Te Deum et relais à toutes les paroisses par les mandements épiscopaux est justement achevée dans les années 1690-1710. Voir M. Fogel, 1989, p. 235-245.
} 
l'usurpation du statut, telles ces bourgeoises que l'édit de 1548 accuse de s'être «faictes damoiselles en un jour» (Fogel, 1987, p. 231). Daniel Vaillancourt pose avec raison la question de savoir comment la ville et ses habitants roturiers peuvent prétendre à la magnificence réservée de droit au roi et à la noblesse. Mais peut-on le suivre lorsqu'il affirme : «La rue devient un théâtre qui légitime les pratiques somptueuses du Roi... C'est un théâtre, car le luxe et la dépense nécessitent un auditoire et, avant la lettre, dans cette rue, s'énonce une société de Cour, prête à jouer et à se représenter. »(p. 51)? On verra plus loin ce que peut apporter la métaphore du théâtre à la compréhension de ces pratiques ostentatoires, mais la question du destinataire est effectivement intéressante. Encore que, dans Le pain et le cirque, Paul Veyne ne croyait pas qu'elle fût nécessairement bonne à penser: "L'ostentation n'est pas une action que le riche exerce sur le spectateur, comme un serpent fascine un oiseau; l'un et l'autre sont fascinés par une tierce chose qui appartient à l'un et pas à l'autre : la richesse et le pouvoir » (p. 68)...

Dans le cas des entrées, il me semble que l'interprétation par la réciprocité du don gardait toute sa pertinence; il suffisait d'y ajouter le contenu même des lois somptuaires qui fait du service du roi, à la guerre comme en temps de paix, un espace particulier de la dépense. Et cela, suivant un modèle royal élaboré, comme l'a montré Jacques Krynen, à la fin du XIV ${ }^{\mathrm{e}}$ siècle en rupture avec l'ascétisme chrétien du roi saint Louis IX, non pour rechercher la légitimation par le peuple, mais au contraire, pour affirmer le caractère indiscutable de son pouvoir.

La citation du dictionnaire de Furetière mise en exergue de l'ouvrage (p. 7) présente la cérémonie comme un «[a]ssemblage de plusieurs actions, pompes \& manières d'agir, qui servent à rendre une chose magnifique \& plus solennelle ». En regard des études précédentes sur la magnificence, celle de Fanny Cosandey permet d'aborder la solennité sous l'angle très révélateur de l'ordre de marche des cortèges d'entrée.

«Donner le rang », c'est-à-dire définir, à partir de la position que le roi occupe dans le segment central signalé par le dais, la juste répartition des hommes qui le servent, nobles et officiers, dépasse la simple technique et relève de l'art de gouverner. D'une part, la place dévolue à 
chaque corps doit correspondre à son importance sur l'échelle des pouvoirs, de même que la place dévolue à chaque individu à l'intérieur de son corps mesure le degré de sa dignité. Mais d'autre part, cette dévolution relève de la seule volonté royale, de la capacité du roi à « or-donner» la multiplicité des rivalités mouvantes.

Fanny Cosandey relie très justement le développement de cette nouvelle fonction royale à la cérémonialisation de tous les actes de la vie du roi qui marque les temps troublés de la deuxième moitié du $\mathrm{XVI}^{\mathrm{e}}$ siècle et que résume l'édit de création de la charge de grand maître des cérémonies en 1585: "faire reconnaittre la dignité et splendeur convenable à notre grandeur » (p. 22).

Si la question du rang se pose dès lors à tout moment et à l'intérieur même des palais royaux, le cortège d'entrée a l'originalité de faire l'exposition solennelle de l'ordre monarchique dans sa totalité devant les habitants de la ville et, par le biais des relations diplomatiques et officielles, devant les autres villes et les cours étrangères. À défaut de déchiffrer les allusions subtiles des décorations, chacun peut voir qui est plus ou moins proche du roi, qui à droite plutôt qu'à gauche. D'autant que les vêtements, par leur qualité et par leurs couleurs, signalent le passage d'un segment, donc d'un corps, à un autre. Ainsi les grands audienciers et contrôleurs généraux demandent-ils à être débarrassés de leurs robes de velours noir à manches pendantes qui les font confondre avec les huissiers, mais se heurtent aux maitres de compte dont ils voudraient prendre les habits. Fanny Cosandey parle d'un jeu de domino où l'on ne peut bouger une pièce sans entraîner l'ensemble.

Pourtant, à l'évidence, malgré les prétentions à l'immuabilité de l'ensemble du système monarchique et bien que le rang tende à devenir un bien patrimonial au même titre que le fief ou l'office qui le justifient, les positions varient : nouveaux corps, nouveaux individus et nouveaux principes multiplient les occasions de désordre et donc, pour le roi, les occasions d'intervenir, d'imposer un ordre qui n'est que la couverture de sourdes querelles. Bien patrimonial pour les particuliers, position dans des rapports de pouvoir dont le roi 
revendique l'entière maittrise, le rang est devenu une affaire trop sérieuse pour être abandonné aux initiatives urbaines.

Avec, parfois, des impasses, comme le montre l'affaire des ducs et pairs au moment de la fameuse entrée de Louis XIV et de MarieThérèse à Paris en 1660. Au siècle précédent, les ducs et pairs ont déjà dû céder la préséance aux princes du sang, les voici menacés de passer après les princes des maisons d'origine étrangère. À l'approche du mariage du roi, ils multiplient les démarches, présentent un mémoire sur leurs droits, s'adressent au roi : celui-ci les fait attendre jusqu'à la veille de l'entrée où, avec un conseil restreint, il revoit l'ensemble du dispositif du cortège et convoque les ducs et pairs pour leur faire entendre sa décision finale le lendemain matin au lieu même où il siègera pour la première partie de l'entrée. Refusant un piège auquel ils ne pourraient échapper publiquement, les ducs et pairs s'abstiennent de figurer dans le cortège. Colère royale et châtiments.

Faut-il pour autant parler de mise en scène? Et d'une manière générale, quelle est l'utilité des références si courantes au théâtre pour notre compréhension du cérémonial?

S'il s'agissait de l'Angleterre, il faudrait sans doute écouter la reine Elizabeth I elle-même dans son adresse aux députés des Lords et des Communes en 1586: «We Princes are on stages in the sight of view of all the World. » Sous son règne, il y a eu jusqu'à huit théâtres à Londres.

Mais à lire Christian Biet sur Le More cruel (p. 287-318) dans Les Jeux de l'échange et dans Qu'est-ce que le théatre?, ainsi que le beau recueil paru en 2001, Les arts du Spectacle au théâtre (voir Frappier et Quéruel), rien de tel ne se passe en France jusqu'aux années 1630-1650. Indistinction des lieux, des salles dans les palais aux «échafauds » avec leur sens et leurs usages multiples; indistinction du vocabulaire, depuis le «théâtre du monde » jusqu'à la « scénographie », technique de dessin attachée à l'architecture, voire à l'art militaire; mise en action des textes qui relève à la fois des usages de la rhétorique et de la collaboration entre l'auteur, le régisseur avec ses accessoires et les comédiens. Quant aux textes eux-mêmes, ils associent généralement pouvoir, violence et 
châtiment, rien qui renvoie au roi une image de maitrise de lui « comme de l'univers », jusqu'à Corneille précisément.

Si au tournant des $\mathrm{XVI}^{\mathrm{e}}-\mathrm{XVII}{ }^{\mathrm{e}}$ siècles, commence à se constituer le théâtre comme nouveau lieu de sociabilité urbaine, et non curiale, il s'y forme un art neuf qui emprunte, dit Christian Biet, « à d'autres arts de la parole, dont celui de la chaire et du barreau, à d'autres arts représentatifs, dont ceux des ballets, des entrées et des divertissements royaux» (p. 285). Voici donc la proposition retournée, et le théâtre débiteur des cérémonies...

Quant aux cérémonies, de quoi peuvent-elles être débitrices si ce n'est en premier lieu du modèle religieux? Ce que disent les dictionnaires contemporains de celui de Furetière. Voyons Richelet (1680) «Cérémonie : Culte extérieur de La Religion [...] Actions \& manieres honnestes qui se font en public par les Princes, les Grands et les Magistrats.» — et l'Académie (1694) — «Cérémonie: Action mystérieuse qui accompagne la solemnité du culte extérieur que l'on rend à Dieu [...] se dit de certaines formalitez qu'on observe dans les actions solemnelles pour les rendre plus éclatantes. »

Référence : Marie-France Wagner, Louise Frappier et Claire Latraverse (dir.), Les jeux de l'échange: entrées solennelles et divertissements du XV $V^{e}$ au XVII siècle, Paris, Honoré Champion, coll. «Études Essais sur la Renaissance », 439 p.

\section{Bibliographie}

BAUDRILLLART, Henri. 1867-1880, Histoire du luxe privé et public depuis l'Antiquité jusqu'à nos jours, Paris.

BIET, Christian et Christophe TRIAU. 2006, Qu'est-ce que le théâtre?, Paris, Gallimard, coll. « Folio-Essais ».

Delamare, Nicolas. 1713-38, Traité de la police, Paris. 
FoGEL, Michèle. 1989, Les cérémonies de l'information dans la France du XVI e au XVIII siècle, Paris, Fayard;

—. 1987, «Modèle d'État et modèle social de dépense : les lois somptuaires en France de 1485 à 1660 », dans Jean-Philippe Genet et Michel Le Mené (dir.), Genèse de l'État moderne. Prélèvement et redistribution, Paris, Éd. du CNRS, 1987, p. 227-235.

FRAPPIER, Louise. 2001, «Rhétorique et théâtralité : aspects de la déclamation dans la tragédie humaniste », dans Marie-France WAGNER et Claire LE BRUN-GouAviC (dir.), Les arts du spectacle au théâtre (1550-1700), Paris, Honoré Champion, p. 35-50.

GuENÉE, Bernard et Françoise LEHOUX. 1968, Les entrées royales françaises de 1328 à 1515, Paris, CNRS.

KRYNEN, Jacques. 1981, Idéal du prince et pouvoir royal à la fin du Moyen Âge (1380-1440). Étude sur la littérature politique du temps, Paris, A. et J. Picard.

LiPOVETSKY, Gilles et Élyette Roux. 2003, Le luxe éternel : De l'âge du sacré au temps des marques, Paris, Gallimard.

NAGLE, Jean. 1998, La civilisation du cour : histoire du sentiment politique en France du XI au XIX siècle, Paris, Fayard.

QUÉRUEL, Danielle. 2001, «"Hystoires" et "personnages" » : quand la rue devient théâtre à la fin du Moyen Âge », dans Marie-France WAGNER et Claire LE BRUN-GOUAVIC (dir.), Les arts du spectacle au théâtre (1550-1700), Paris, Honoré Champion, p. 37-60.

VEYNE, Paul. 1976, Le pain et le cirque. Sociologie historique d'un pluralisme politique, Paris, Seuil.

ZEMON DAVIS, Natalie. 2003 [2000], L'esprit du don dans la France $d u X V I^{e}$ siècle, Paris, Seuil. 